\title{
Déchiffrer Vénus. \\ Renaissance et Âge classique
}

\author{
Daniel Droixhe
}

Au chapitre de la Nature des dieux traitant de l'étymologie de leur nom, Cicéron mentionne pour terminer comme s'il s'acquittait d'une explication moins convaincante - celui de Vénus, « ainsi appelée par notre nation parce qu'elle est la déesse qui vient à toute chose » : ad res omnes veniret. "Son nom », ajoute-t-il, "n'est pas dérivé du mot venustas [beauté] mais plutôt venustas d'elle ${ }^{1} »$.

Une autre explication apparaît dans la Cité de Dieu, au passage où saint Augustin se moque des interprétations "obscènes" alléguées par les "païens lettrés [...] en défense de leurs dieux $^{2} »$. Se composer une divinité pour la nourriture (Educa) et une autre pour la boisson (Potina), "n'est-ce pas plus convenable à la bouffonnerie des mimes qu'à la dignité des dieux ? ». Le dieu Liber " libère » les mâles de leur semence. On lui dédie en conséquence, dans son temple, des organes masculins, comme on voue des organes féminins à Libera. Sur le coït veillent plusieurs protections :

quand un couple de mariés croit que tant de dieux des deux sexes sont présents et participent à l'opération, n'est-il pas pris de honte au point qu'il perd son ardeur et qu'elle augmente sa résistance?

Pour la «tâche » amoureuse, « un seul dieu ou déesse ne serait-il pas suffisant »? " Vénus seule ne serait-elle pas sans pareille à l'occasion » ? On dit en effet que « son nom provient du fait que, sans violence, une femme ne cesse d'être une vierge » - conception jouant sur le rapport entre Vénus et sine vi. Mais d'autres figures mythologiques réclament l'abandon de la femme au désir masculin. Il y a un dieu pour défaire sa ceinture (Virginensis), un autre pour la « tenir en bas » dans l'amour, "faible et terrorisée », " pour qu'elle perde sans difficulté sa virginité » (Prema), et un autre dieu encore (Subigus) pour qu'elle soit soumise à son mari.

\section{La Renaissance et la redécouverte du Livre des Rois}

La Renaissance allait apporter son lot d'interprétations nouvelles, principalement suscitées par la lecture ou la redécouverte de textes antiques.

Joseph Juste Scaliger, en son commentaire de la Signification des mots de Festus (1584), inscrit son hypothèse dans la tradition cicéronienne d'explication paronymique. "Vénus est une divinité importée, mais son nom est romain : il signifie "étranger". En effet, il est fait de veniendo [...] ». Un autre texte va polariser l'interrogation et l'articuler progressivement à la recherche sur de nouveaux secteurs de la philologie ou des sciences humaines. Le deuxième

\footnotetext{
${ }^{1}$ Cicéron, De natura deorum, éd. Loeb (268), Cambridge, Harvard U.P., 1979, p. 89.

2 Saint Augustin, Euvres, 33-37. La cité de Dieu, éd. G. Bardy et G. Combès, Paris, Desclée de Brouwer, 1980, livre VI, chap. viii-ix ; éd. Loeb, II, p. 333
} 
Livre des Rois raconte comment le souverain d'Assyrie fit venir en Samarie divers peuples pour prendre la place des Hébreux, oublieux du vrai Dieu. Mais ces populations se refusèrent à suivre la loi d'Elohim et restèrent prisonnières de l'idolâtrie :

En fait, chaque nation se fit son dieu et le plaça dans les maisons des hauts lieux, que les Samaritains avaient construites. Chacune des nations agit ainsi dans les villes où elle résidait : les gens de Babylone firent un Soukkoth-Benoth; ceux de Kouth, un Nergal; ceux de Hamath, une Ashima; les Awites, un Nibhaz et un Tartaq [... $]^{3}$.

Cette expression de Soukkoth-Benoth, ou Succoth-Benoth, a fait couler beaucoup d'encre. Passons sur les interprétations de la critique moderne ${ }^{4}$. La tradition interprétait le premier terme, fréquent dans la Bible, comme signifiant "tente, cabane, abri ». La Genèse appelait Sukkoth un endroit où Jacob avait fait des « abris » pour le bétail. Les Septante avaient donc traduit le mot par skênê « cabane, hutte ». Le deuxième élément de la formule semblait quant à lui se rattacher à bén " enfant, jeune ». Ainsi, Soukkoth-Benoth fut généralement expliqué en latin par tabernacula puellarum «cabanes de jeunes». Mais les traductions des Écritures durent se borner à reproduire telle qu'elle l'expression, que le contexte maintenait dans une grande obscurité.

La tradition rabbinique, de manière à peine moins énigmatique, l'interprétait assez largement comme renvoyant à l'image d'une poule avec ses poussins. C'est ce qu'on trouve au Moyen Âge chez Rachi, auteur du « commentaire sur la Tora le plus populaire de toute la littérature exégétique juive ${ }^{5}$ », et chez David Kimhi. Comme l'écrit celui-ci :

Nos rabbins, d'heureuse mémoire, disent que Succoth Benoth était une poule, comme si l'on disait que les Assyriens honoraient l'image d'une poule appelée Succoth Benoth. C'est qu'on nomme cet animal Suecui, signifiant littéralement « celle qui couvre »; ils appellent ainsi les poules Succoth, quasiment "celles qui couvent et couvrent», et expliquent Benoth comme désignant ses petits, qu'elles ont coutume de couver et de couvrir de leurs ailes ${ }^{6}$.

En outre, les rabbins soulignent le rapport unissant cette attitude protectrice et le sens général de « tente, abri ».

Certains commentateurs, il est vrai, prétendirent voir dans Soukkoth-Benoth, comme dans les termes voisins de Nergal, Ashima ou Nibhaz, des noms de villes. La mise au point était venue, au début du IVe siècle, de saint Eucher, évêque de Lyon, qui écrivit dans ses Commentaires sur la Genèse et le livre des Rois :

\footnotetext{
${ }^{3}$ Rois 2, 17/30.

${ }^{4}$ Karel van der Toorn et al., Dictionary of deities and demons in the Bible, Leyde, Brill, 1995, p. 1553-1556. La critique moderne y a souvent reconnu le nom déformé de la déesse Zarpanit(u), épouse de Marduk, le dieu-patron de Babylone.

${ }^{5}$ Salomon ben Isaac (dit Rasi), né à Troyes vers 1040, mort en 1105. Cf. Salomon Ben Isaac, Le commentaire de Rachi sur le Pentateuque, trad. fr. par I. Salzer et al., Paris, Comptoir du livre du Kéren Hasefer, 1957, préface.

${ }^{6}$ D'après Athanasius Kircher, Oedipus aegyptiacus, Roma, ex typogr. V. Mascardi, 3 tomes, 1652-1654, p. 354.
} 
Il me semble cependant que les noms en question ne puissent se comprendre, en vertu de la liaison du discours [c'est-à-dire du contexte] que comme ceux d'idoles auxquelles ces peuples étaient assujettis $[\ldots]^{7}$.

\section{L'hypothèse de Selden}

On en était là lorsqu'intervint John Selden ${ }^{8}$. Son traité Sur les dieux assyriens fit en 1617 forte impression. On a parfois dit qu'il avait cédé au «préjugé courant en faveur de l'antiquité de l'hébreu ». Plus exactement, il déplace la priorité historique vers cette Assyrie babylonienne ou babélique dans laquelle Joseph Scaliger avait désigné le vrai berceau du monde sémitique et une alternative à l'exorbitant privilège que le judaïsme faisait peser sur l'histoire de l'humanité. Selden mit en évidence et développa le rapport unissant l'expression SoukkothBenoth et un passage de Valère Maxime dans ses Faits et dits mémorables ${ }^{9}$. Il y est question d'un temple établi dans la colonie phénicienne de Sicca, qu'on identifie aujourd'hui avec El Kef en Tunisie, où les femmes puniques manifestent toute «la laideur de leur conduite honteuse » :

En effet il y a à Sicca un temple de Vénus où les femmes d'âge nubile se rendaient et, partant de là, elles amassaient l'argent de leur dot au moyen des injures auxquelles leur corps était soumis, décidées à s'engager dans des liens honnêtes par le biais de liaisons si déshonorantes.

Ce lieu de débauche sacrée doit évidemment être identifié, pour Selden, avec les tabernacula puellarum des Écritures, et Benoth est sans aucun doute la forme originelle du nom de Vénus. L'auteur anglais, et d'autres à sa suite, rappelleront que l'ancienne Sicca, volontiers nommée Sicca Venerea, était célèbre parmi les Anciens: Ptolémée, Pline, Procope, l'Itinéraire d'Antonin en font mention. Victor d'Utique, qui en parle dans sa Persécution des Vandales, en était originaire, de même qu'Arnobe l'Ancien. Si ce dernier ne mentionne pas le temple de Vénus dans son traité Contre les Gentils (fin du IIIe siècle), c'est sans doute que ce maître d'école n'était pas trop fier de la réputation de sa ville ${ }^{10}$.

\footnotetext{
${ }^{7}$ Eucher, Commentarii in Genesim et in libres Regum, Rome, Apud Paulum Manutium, 1564, p. 297.

${ }^{8}$ Cf. David Sandler Berkowitz, John Selden's formative years. Politics and Society in early seventeenth-century England, Washington, The Folger Shakespeare Library, 1988.

${ }^{9}$ Saint Augustin, Euvres..., op. cit., livre II, chap. 6/15 ; Maxime Valère, Faits et dits mémorables, éd. R. Combès, Coll. « Budé », t. I, Livres I-III, 1995, p. 186 ; John Selden, De dus syris syntagmata I, Londres, Bibliopolarum corpori excudebat Guilielmus Stansbeius, 1619, chap.Vl, p. 216 sq.

${ }^{10}$ Arnobe l'ancien, Contre les Gentils, éd. H. Le Bonniec, Coll. «Budé », 1982, 36/4, p. 161 ; commentaire sur Sicca, p. 8. Arnobe attaque notamment le culte de Vénus dans un passage ridiculisant les dieux païens susceptibles de reprocher à un chrétien d'adorer un «homme mort vilement sur la croix ». Le grief viendra-t-il d'une déesse «courtisane prostituant ses charmes secrets »? Un passage de Léon l'Africain (Description de l'Afrique, éd. A. Épaulard, Paris, Maisonneuve, 1981, I, 217) aurait pu faire attendre un rapprochement supplémentaire avec Soukkoth. Le chapitre sur les Devins officiant à Fez (livre III) évoque les moeurs de « femmes qui font croire au peuple qu'elles sont liées d'amitié avec certains démons ». Ceux-ci sont invoqués « avec une grande déférence ». " Mais les gens qui joignent à l'honnêteté une certaine instruction ainsi que l'expérience des choses nomment ces femmes Sahacat, ce qui a le sens du mot latin fricatrices. Et en vérité, elles ont cette maudite habitude d'user l'une de l'autre, ce que je ne peux exprimer par un terme plus décent. Lorsqu'il se trouve une belle femme parmi celles qui viennent les consulter, elles s'en éprennent ainsi qu'un jeune homme s'éprend d'une jeune fille et, comme si le démon parlait en personne, elles lui demandent en paiement des embrassements amoureux. La femme, qui croit devoir complaire à l'esprit, y consent le plus souvent. »
} 
Le caractère relativement récent du nom de la déesse plaidait en faveur de l'hypothèse assyrienne. "Dans les premiers temps de Rome, sous les rois, Vénus était tout à fait inconnue. » On n'en fait pas mention dans les chants Saliens, à la différence d'autres divinités. Varron, discutant dans la Langue latine l'origine des noms des mois, contestait qu'avril vienne d'Aphrodite, c'est-à-dire de Vénus : «je n'ai rencontré son nom nulle part dans les anciens textes $^{11} »$. Macrobe, dans les Saturnales ${ }^{12}$, rapportait de son côté la notation d'un certain Cingius qui, dans ce qui subsiste de son livre De fastis, observait que « les Anciens n'ont institué nulle fête et nul sacrifice insigne en l'honneur de Vénus pendant le mois d'avril ».

Si l'étymologie à partir d'Aphrodite et Vénus avait été proposée « à la légère », celle rattachant la divinité romaine à veniendo, argumentait Selden, n'était pas plus convaincante. « Les Latins auraient à coup sûr observé une analogie plus soigneuse et n'auraient pas formé Vénus au moyen d'une terminaison masculine, comme on voit qu'ils s'en abstiennent dans tous les autres noms de déesses. »

À côté de la morphologie ancienne, la phonétique doit être prise en compte pour raisonner sur la filiation Benoth > Vénus :

Il n'est personne qui ne sache que le changement de la lettre $B$ en $V$ est très facile et très fréquent, et si, d'autre part, on prononce le tau final comme un sigma, ainsi que font les juifs d'aujourd'hui, on obtiendra, de la manière la plus nette, le mot Venos pour Benoth : et Binos, prononcé à la grecque, est notre Vénus ${ }^{13}$.

« Je n'oserais pas », conclut Selden, « assurer à cent pour cent mon étymologie », bien qu'il soit notoire que certains mots italiques viennent de la Syrie « ou de son voisinage ». Pourtant, toutes les autres suppositions - qu'elles soient dues à Cicéron, Augustin ou Scaliger ne paraissent, à côté, « d'aucun prix ».

\section{Du signe linguistique au signe zodiacal : Kircher}

La démonstration de Selden trouva bientôt des partisans qui placèrent Vénus dans le cadre d'une mythologie comparée ou d'une lecture des astres. Gérard Vossius déclina les autres noms de la divinité dans sa Théologie des gentils de $1641^{14}$. Parmi ceux-ci figuraient Salambô la Babylonienne et Baaltis, sœur d'Astarté — figures de la lune ou de maîtresses du ciel.

\footnotetext{
${ }^{11}$ Varron, 1970, 33, p. 18 : « je pense qu'Aprilis a plutôt été ainsi nommé parce que le printemps ouvre [aperit] tout».

${ }^{12}$ Macrobe, Opera, 2e éd., Leipzig, Teubner, 1970, 1, 12, 12, p. 12-13. L'opinion de Cingius est ici rapportée de manière contradictoire, car, selon Varron, il était partisan de la filiation Aphrodite > avril. Sur l'identité de cet auteur, voir les notes de P. Flobert dans l'édition de Varron (La langue latine, Livre VI, éd. P. Flobert, Coll. « Budé », 1985, 110) qui énumère les intervenants dans la question de l'origine d'aprilis, dont Ovide, Plutarque, Virgile (partisan d'aperire), Isidore de Séville (partisan d'Aphrodite). On y remarque aussi « que la fête de Venus Verticordia tombe le 1er avril ».

${ }^{13}$ John Selden, De dus..., op. cit., p. 220.

${ }^{14}$ Vossius 1641, chap. 22, p. 209. Johann Heinrich Ursin s'y référa en 1658 dans des Analectes sacrées.
} 
En 1652, dans son Edipe égyptien, Athanase Kircher confronte les deux interprétations de Succoth Benoth comme " temple ou lieu sacré », selon Selden, ou comme symbole de la poule accompagnée de ses poussins, selon les Hébreux ${ }^{15}$. Il suppute que les noms des idoles fabriquées par les nouveaux peuples ayant pris la place des Hébreux devaient renvoyer aux astres, conformément à une pratique archaïque courante. Or, les Pléiades, chez les Égyptiens, « étaient désignées sous le nom de Poule » parce que les sept étoiles « offrent l'image d'une poule avec ses poussins » :

S'y ajoutait le rapport à Vénus, vue comme un animal accompagné de ses petits, dans la mesure où elle couve de son humeur et de sa chaleur féconde les êtres d'ici-bas et les nourrit. Enfin, la poule est un animal lascif, prolifique.

Telle est l'explication du nom de Succoth Benoth que portait Vénus chez les Assyriens. Elias Schedius, dans ses Dieux germaniques de $1648^{16}$, appuiera l'argument par une mise en évidence de la cognatio des sons $b$ et $v$, à partir d'une leçon du Soldat fanfaron de Plaute ${ }^{17}$. On y trouve exuiuit pour exbibuit " il a bu ». Quant à la transformation de la finale du nom assyrien de Vénus, elle est appuyée par une des Monnaies des empereurs romains décrites par Adolf Occo dans un ouvrage sorti en 1579 des presses anversoises de Christophe Plantin ${ }^{18}$. On y lit le nom de Julia Augusta, épouse de Septime Sévère (fin du IIe siècle), et au revers l'inscription VENOS GEN. s.c. ${ }^{19}$.

L'hypothèse de Kircher ne fut cependant pas unanimement acceptée. Un certain Johann Frischmuth, demeuré particulièrement obscur, présenta en 1663 à l'université d'Iéna, sous la direction de son patron Salomon König, une dissertation dans laquelle il invoquait les anciens

\footnotetext{
${ }^{15}$ T. I, synt. IV. Pantheon Hebraeorum, chap. 18, p. 354-360.

16 Elias Schede, De dis germanis, sive Veteri Germanorum, Gallorum, Britannorum, Vandalorum religione syngrammata quatuor, Amsterdam, Apud Ludovicum Elzevirium, 1648, p. 122-123.

${ }^{17}$ Plaute, Comoediae, Accedunt commentarii Fr. Taubmanni auctories, s.l., Apud Zachariam Schurerum, 1621, p. 845 ; Plaute, [Comédies], éd. A. Ernout, Coll. « Budé », t. I et IV, 1989-1990, IV, p. 228 ; sur les mss du Miles gloriosus, $c f$. l'introduction d'A. Ernout dans Plaute, ibid., I, p. xxvii-xxviii. Schedius mentionne l'édition de Plaute donnée par Frédéric Taubmann, qui se fonde lui-même sur les manuscrits " palatins » et en particulier sur le Codex vetus Camerarii rendu célèbre par l'utilisation qu'en fit au milieu du XVIe siècle Joachim Kammermeister, l'ami de Dürer (manuscrit aujourd'hui à la Vaticane).

${ }^{18}$ Corp. Inscr. Lat. 1564.
}

D.M.

Claudiæ Veneri

ae. Coniugi. Sanctiss

imae. Q iusteius. Probatus. sibi. suis

que. porterisque

eorum

${ }^{19}$ Adolf Occo, Impp. Romanorum numismata, Anvers, Ex off. C. Plantini, 1579, p. 253. La monnaie appartient à la série où l'impératrice est désignée par l'expression IULIA PIA FELIX AUG. Le nom et l'image de la VENVS GENETRIX, par ailleurs, figurent sur plusieurs de ses médailles : cf. H. Cohen, Description historique des monnaies frappées sous l'empire romain, 2e éd. Réimpr. anast., Maestricht, A.G. van der Dussen, 1983, IV, p. 123, qui ne mentionne pas d'exemplaire avec VENOS, mais plusieurs autres se rapprochant de celle invoquée par Selden (voir les $n^{\circ} 203$ sq.). 
R. Salomo et R. Abarbenel pour prendre ses distances avec la thèse du jésuite et discerner plutôt sous la figure de Vénus la reine de Saba, « souveraine du ciel ${ }^{20}$ ».

\section{Du signe zodiacal à la figure érotique : Vénus et Europe}

Une autre figure zodiacale se surimposait au «rapport» entre l'image de la poule accompagnée de sa progéniture et Vénus. Sous le signe du taureau, qui domine, au printemps, la terre, poursuivait Kircher, "se pare d'un vêtement verdoyant, comme si elle donnait à regarder aux mortels le spectacle d'une végétation luxuriante où s'exprime je ne sais quelle lubricité intérieure ». C'est alors que "les oiseaux et tous les autres animaux, agités des démangeaisons de Vénus, songent à s'unir pour proliférer». Le taureau incarnait cette « lubricité » sous la traduction mythologique de Jupiter procédant à l'enlèvement d'Europe. Un « jeu d'échange», comme dit Josephine Hildebrand (1988), s'instaure entre les deux personnages dans la peinture de l'âge classique. Simon Vouet traite de la même manière, vers 1641-1642, l'Enlèvement d'Europe et l'une de ses Toilettes de Vénus. La première divinité, assise sur le taureau Jupiter comme sur un lit de parade, évoque la position de Vénus dans l'autre tableau; de part et d'autre, une ou plusieurs jeunes filles sont occupées à la toilette de la déesse, Europe apparaissant ici comme la future épouse que l'on couronne et enguirlande sous le regard des amours, toute crainte évacuée, etc.

Le réseau mythologique étend ainsi vers le couple Jupiter-Europe l'illustration des dangers ou dévoiements du désir. Le dieu y révèle sous le signe taurin sa nature de " sale animal » et Europe, vierge imprudente qui le «flattait de la main", devient le symbole des dangers menaçant ceux qui, comme l'écrit Noël Conti dans sa classique Mythologie, « suivent leurs appétits et concupiscence charnelle ». Boccace, dans sa Généalogie des dieux, avait qualifié Jupiter d'« entremetteur », puisque le «taureau blanc » de la fable ne faisait, selon lui, que déguiser la prosaïque réalité d'un vaisseau voué à la traite des femmes ${ }^{21}$. Le décor démultipliait ce symbolisme de base en confirmant l'hypothèse philologique de Selden sur le nom «syrien » de Vénus. Europe n'était-elle pas fille du roi de Phénicie ? Dans le Roman de Leucippé et Clitophon, Achille Tatius, écrivain alexandrin des IIIe-IVe siècles, avait dépeint l'enlèvement d'Europe dans un paysage « pouvant être reconnu comme celui des habitants de Sidon $^{22} »$.

Un dispositif imaginaire très homogène réunissait désormais la Vénus " obscène » de saint Augustin, les « tabernacles de jeunes filles », l'image de la brûlante Afrique et particulièrement la Phénicie adonnée au culte de Priape. N'avait-on pas avec raison, avait

\footnotetext{
${ }^{20}$ Johann Frischemuth et Salomon König, [...] sive de Melechet Coeli exercitium philologicum, Iena, Typis Samuelis Krebsii, 1663, chap. I, § 1-2.

${ }^{21}$ Boccace, Genealogie, In vico divi Jacobi, sub intersigniis trium coronatum coloniensium atque divi Martini, éd. Johann Kierher, Paris, 1511, fo XXI.

${ }^{22}$ Passage reproduit par Johann Heinrich Ursin, Analectorum rhetoricorum libri duo, Nuremberg, Typis Mich. Endteri, 166o, p. 371 sq. Conti Natale, Mythologie, ou explication des fables, Paris, Pierre Chevalier, 1627, p. 939-943, souligne cette origine par l'étymologie. Cadmus, chargé de retrouver sa soeur après l'enlèvement, fait la rencontre d'un animal sacré « près de la fontaine de Thurie (ainsi nommée de Thur, qui en langue Phoenicienne signifie une vache) ».
} 
conclu Selden, surnommé jadis Vénus la " persane », terme qui « désigne particulièrement, comme les doctes le savent, les Babyloniens et Assyriens »? Ajoutons-y l'observation philologique concernant le mot de phénicianiser, définissant chez Lucien ou Galien, une des pratiques les plus « infâmes » parmi celles qu'a suscitées la luxure ${ }^{23}$ ?

\section{Du signe linguistique au signe ethnique}

La figure de Vénus ne manquait pas au panthéon germanique. Olaus Magnus avait évoqué, dans son Histoire des peuples du Nord publiée à Rome en 1555, la déesse qui « manifeste la turpitude du sexe » : "Frea ou Friga est honorée chez les Goths comme Vénus chez les Romains. Le vendredi lui est resté consacré jusqu'aujourd'hui ${ }^{24}$. » Le rapprochement s'impose d'autant plus qu'elle est " femme d'Odin, ou de Wodan, assimilé à Mars », ajoute Ole Worm dans ses Fastes danois $(1636)^{25}$.

Mais d'où vient donc ce nom de Frea, et que signifie-t-il qui ait pu laisser une trace vivante dans la langue et la culture germaniques? Dans une lettre de 1651 qui sera imprimée sous le titre À propos de Frea (Vénus), Wodan (Mars) ou Odin, Christoph Arnold développe une argumentation linguistique exemplaire. Il se fonde d'abord sur les « codes barbares » - lois des Lombards et autres — dont les Bohier, les Herold, les Pithou avaient entrepris puis perfectionné l'édition depuis le début du XVIe siècle. On y trouve souvent le mot de frea, par exemple dans une loi lombarde du roi Limprand condamnant « toute personne qui a l'audace de faire sortir de sa maison, contre son gré, une frea dépendant d'autrui ${ }^{26}{ }^{»}$. Les grands spécialistes de ces textes, comme Friedrich Lindenbrog dans son Code des anciennes lois de 1613 ou Henry Spelman dans son Archéologue en forme de glossaire de 1626, se sont interrogés sur le sens du terme :

Je pense [dit Spelman] qu'il s'agit d'une jeune fille sans parents, mineure ou orpheline, vu qu'elle est dite in mundio, ce qui signifie sous tutelle, ou sous la puissance d'un autre.

En effet, explique Christophe Arnold, ce terme de mundius offre manifestement un rapport avec notre allemand Vormund «tuteur», ce dernier étant littéralement «celui qui se tient devant la personne appelée mundo ». On peut aussi invoquer l'anthroponymie. Le prénom Edmundus a été interprété par Richard Verstegan, dans ses Restitution of decayed intelligence, comme un composé du saxon ed "serment» et d'une forme de muth, c'est-à-dire mouth «bouche », demeuré mond " en Belgique ». Arnold possède un exemplaire de ce "livre

\footnotetext{
${ }^{23}$ Friedrich-Karl Forberg, Manuel d'érotologie classique, (1966), trad. Isidore Lisieux, Paris, postface et note de Pascal Pia, éd. Joëlle Losfeld, Paris, 1995, chap. 3. De l'irrumation, p. 87 sq.

${ }^{24}$ Magnus Olaus, Historia de gentibus septentrionalibus, Rome, Apud Ioannem Mariam de Viottis Description of the Northern peoples, transl. by P. Fisher \& H. Higgens, Rome, 1555, 1. III, chap. 3.; éd. P. Foote, London, The Hakluyt Soc. 1996-1998.

${ }^{25}$ Ole Worm, Fasti danici, Copenhague, apud J. Moltkenium, 1643, l. I, chap. 15.

${ }^{26}$ Christoph Arnold, [lettre du 16 déc. 1651]. De Frea (Venere), Wodano (Marte) sive Othnio, et diversa scriptione de libera puella, dans Georg Richter, Epistolae selectiores, 1662,1. II, titre 46.
} 
élégant » où le Hollandais Johannes De Laet, bien connu en histoire de la linguistique, a noté : «Mund est le patron, le tuteur. »

Le mot de Frea, apparenté à l'allemand frei, à l'anglais free, porte par ailleurs une incontestable idée de liberté :

De même, chez les Flamands, un adolescent libre est dit Vryder, ou Vryer; chez les anciens Germains, c'était Frank pour un garçon, ce qui est fry Ank, et Vrydster, Fryster ou même autrefois Vryersse pour une fille. Ceci convient en tout point avec le mot persan Fristar, vierge, comme Marnix de Sainte-Aldegonde l'a montré voici bien longtemps à partir du lexique de Raphelengius, que celui-ci a recueilli de son Pentateuque.

Ces quelques lignes valent un résumé cavalier de l'observation séminale ayant stimulé les débuts du "paléo-comparatisme ». Si frea paraît ainsi avoir le sens de " fille sous tutelle », reste la question de savoir à quel statut réel correspondait le mot. Vossius, rappelle Christoph Arnold, se demande si l'on désigne par là une jeune fille non mariée ou celle qui est, par ses parents, de condition libre. C'est dans un autre sens que le nom de la "Vénus des Goths », représenté par les formes Frea, Fria, Frigga ou Fricco, passera selon Arnold dans le langage courant. Le mot est en effet devenu " appellatif » pour désigner une « mère de famille » : on prodigue volontiers le nom de Vénus à celle qu'on aime. Nombre d'écrivains germaniques en témoignent : Heinrich von Presla, Rudolf von Rotenburg, Wolfram von Eschenbach... On peut dès lors penser que l'allemand Frau, comme le nom de l'ancienne déesse, trouve sa " première origine dans freo ou frey, c'est-à-dire l'idée de liberté ». Sans doute est-ce à la même famille de mots qu'il faut rattacher l'allemand freien « épouser » ou le flamand vroedmoeder "sagefemme ».

Conformément à une orientation privilégiée de la philologie allemande, la mythologie s'ouvre d'emblée sur le folklore ou une amorce d'ethnologie pour assurer l'hypothèse. Chez les Scandinaves comme chez les Germains et les Saxons, le vendredi est désigné par la même racine : «Fredagh, Friday, Freitag ». De même que les Romains d'autrefois prêtaient à Vénus un sexe indéterminé, ou les deux genres, ainsi qu'en témoigne Macrobe, une incertitude similaire caractérise la Vénus gothique, également dénommée Frea ou Fricco, avec terminaison masculine. Olaus Magnus écrit : «On la représentait avec des armes, le glaive et l'arc, parce que, dans ces régions, l'un et l'autre sexe était enclin aux armes. » Richard Verstegan, ou Rowlands, la montre également tenant le glaive et l'arc, « ce qui signifie que les femmes, aussi bien que les hommes, devaient être préparées au combat en vue d'une époque de nécessité, d'une situation critique d'adversité ${ }^{27}$ ». Tous ne seront pas complètement convaincus. Jean Georges Keyssler associera Freia ou Frigga à Frejus, divinité que mentionnent les vieux écrits scandinaves et qui « est la même chose que le soleil » (Antiquités septentrionales et celtiques, 1720, Dissertation sur le culte du soleil, de Frejus et d'Odin, $1728)^{28}$.

\footnotetext{
${ }^{27}$ Richard (ou Rowlands) Verstegan, Restitution of decayed intelligence in antiquities concerning the most noble and renowned English nation, London, printed for Samuel Mearne, John Martyn and Henry Herringman, Florence, Sansoni, 1673, p. 76.

${ }^{28}$ Johann Georg Keyssler, Dissertatio de cultu salis, Freji et Othini, Halle, Apud Ern. Gottl. Crugium, 1728, chap. 10 et 11, p. 773. On lit par exemple dans la Saga de Hervarar éditée en 1666 par Olof Verelius, titulaire de
} 
On a voulu montrer ici de quelle manière le déchiffrement d'un signe linguistique privilégié croise d'autres codes ou champs de la représentation entre Renaissance et Age classique: code mythologique, signes du zodiaque, monnaie, thématique picturale ou idéologie nationale. De grands contrastes entre les traditions érudites de différents pays s'y dessinent. La vogue des « origines assyriennes », parfois dressées contre le privilège hébraïque, voire judaïque, cède la place à une puissante valorisation du modèle germanique, selon une dynamique d'affrontement décrite par Maurice Olender ${ }^{29}$. En même temps, la réflexion sur Frea laisse apercevoir le prélude à l'efflorescence d'une herméneutique romantique associant " culture populaire » et linguistique. L'idée d'une présence sans cesse activée du passé et de ses «racines primordiales » dans la parole moderne y jouera un rôle central, comme le montrent les écrits de Leibniz ou de son disciple Eckhart à propos du fameux pilier des nautes parisiens, découvert au début du XVIIe siècle ${ }^{30}$.

D'autres travaux, consacrés à Giambullari ${ }^{31}$, Aldrete $^{32}$, Henri Estienne ${ }^{33}$, Grotius ${ }^{34}$, Vico, Hervás y Panduro ${ }^{35}$, Proudhon ou Nietzsche ${ }^{36}$, plaideraient en faveur d'une inscription de l'étymologie dans l'histoire d'une philologie conçue comme «associée aux grands mouvements de la pensée, tant dans les arts et les lettres que dans l'évolution des sciences ou de la philosophie », en fonction de l'interaction unissant « le savoir philologique - savoir sur les textes — et les discours savants — les textes du savoir » (E. Bury) $)^{37}$.

la chaire d'antiquités suédoises à Uppsala, que le roi Heidrich « offrit un grand sacrifice à Frigga, divinité qu'il honorait avant toute autre ». Olaus Verelius , éd. Hervarar Saga, Uppsala, H. Curio, 1672, chap. 14, 39 et 138.

${ }^{29}$ Maurice Olender, Les langues du paradis. Aryens et Sémites: un couple providentiel, Paris, Gallimard/Le Seuil, 1994. Celui-ci et Yves Hersant m'ont permis de soumettre une ébauche de la présente étude au Centre Europe de l'École des Hautes Études en Sciences Sociales ; qu'ils en soient remerciés.

${ }^{30} \mathrm{D}$. Droixhe, L'étymon des dieux. Mythologie gauloise, linguistique et archéologie è l'âge classique, Genève, Droz, 2004.

${ }^{31}$ Eugenio Coseriu, « Las étimologías de Giambullari », Homenaje a A. Tovar, Madrid, Gredos, 1972, p. 95-103 ; Vanwelkenhuyzen 2004.

${ }^{32}$ Lucia Binotti, La teoría del'castellano primitivo'. Nacionalismo y reflexión lingüística en el Renacimento espanol, Münster, Nodus, 1996.

${ }^{33}$ Colette Demaizière, « Les réflexions étymologiques d'Henri Estienne. De la Conformité aux Hypomnèses (1565-1582) », dans Discours étymologiques, Actes du colloque international organisé à l'occasion du centenaire de la naissance de W. von Wartburg, Tübingen, Niemeyer, 1991, p. 201-210.

${ }^{34}$ Giuliano Gliozzi, Adamo e il nuovo mondo, Florence, Nuova Italia, 1977; sur l'utilisation de la linguistique dans la controverse sur l'origine des Américains, voir D. Droixhe, « Mas ignorante que hereje. De Laet, Acosta et l'origine linguistique des Américains », dans Kontinuität und Innovation. Studien zur Geschichte der romanischen Sprachforschung vom 17. bis zum 19.Jahrhundert. Festschrift f. W. Bahner zum 70. Geburtst, éd. G. Hassler \& J. Storost, Münster, Nodus, 1997, p. 73-88 ; sur les rapports entre linguistique et droit, voir R.H. Bloch, Étymologie et généalogie. Une anthropologie littéraire du Moyen Âge français, Paris, Seuil, 1989.

${ }^{35}$ Graziella Tonfoni, « Problemi di teoria linguistica nell'opera di Hervás y Panduro », Lingua e stile 23, 1988, p. 365-381.

${ }^{36}$ Giuliano Campioni, Sulla strada di Nietzsche, Pisa, ETS ed., 1993.

34. ${ }^{37}$ Rapport sur le séminaire " Pour une histoire de la philologie », dans le cadre de l'équipe "États Société Religions en Europe (Moyen Âge/Temps modernes) »(EA 2449). D'une façon générale, voir les travaux de Pascale Hummel, notamment ceux présentés dans ce séminaire [Histoire de l'histoire de la philologie: étude d'un genre épistémologique et bibliographique, Genève, Droz, coll. " Histoire des idées et histoire littéraire 385 ", 2000; Moeurs érudites: essai sur la micrologie littéraire (Allemagne, XVIe-XVIIIe siècles), Genève, Droz, coll. « Histoire des idées et histoire littéraire 395 », 2002]. Sur l'étymologie et les nationalismes linguistiques à la Renaissance, voir J.-C. Margolin, « Science et nationalismes linguistique ou la bataille pour l'étymologie au 
XVIe siècle ", The fairest flower. The emergence of linguistic national consciousness in Renaissance Europe, Florence, Presso l'Accademia, 1991. L'édition et le commentaire philo-logique des anciens textes littéraires français, dans la période de la " préhistoire du romanisme ", mériteraient une attention particulière. Voir Paul Zumthor, "Archaïsme et fiction: les plus anciens documents de la langue romane ", dans La linguistique fantastique, éd. S. Auroux et al., Paris, Denoël, 1985, p. 285-293 ; Brigitte Schlieben-Lange, éd. Frühgeschichte der romanischen Philologie, Tübingen arr., 1987. Une amicale communication de Katelijne Schiltz (Katholieke Universiteit Leuven, Department of Musicology) permet d'ajouter une post-face à la question étymologique posée par Vénus. Celle-ci est associée à Venice (Venise) dans un poème de James Howell qui fait partie de la collection S.P..Q.V, a Survey), of the Signorie of Venice (Londres, 1651). Bercée par des eaux d'une "pureté virginale », ayant conservé son « port de jeune fille », la cité « enchante tous ceux qu'elle a une fois embrassés » : Venus and Venice are Great Queens in their degree,

Venus is Queen of Love, Venice of Policie. 